\title{
Enjeux et spécificités de la recherche en santé animale
}

\author{
C. DUCROT'1, B. BED'HOM²,3, V. BERINGUE4, J.-B. COULON 5 , C. FOURICHON6,7, J.-L. GUERIN 8 '9, \\ S. KREBS ${ }^{6,7}$, P. RAINARD ${ }^{10}$, P. SARRADIN ${ }^{11}$, I. SCHWARTZ ${ }^{4}$, D. TORNY12, M.-L VAYSSIER-TAUSSAT ${ }^{13}$, \\ S. ZIENTARA ${ }^{14,15}$, E. ZUNDEL 16 \\ ${ }^{1}$ INRA, UR346 Epidémiologie Animale, F-63122 Saint-Genès Champanelle, France \\ 2 INRA, UMR1313 Génétique Animale et Biologie Intégrative, F-78352 Jouy-en-Josas, France \\ ${ }^{3}$ AgroParisTech, Génétique Animale et Biologie Intégrative, 16 rue Claude Bernard, F-75231 Paris, France \\ ${ }^{4}$ INRA, UR892 Virologie et Immunologie Moléculaires, F-78352 Jouy-en-Josas, France \\ 5 INRA, Département PHASE, F-63122 Saint-Genès Champanelle, France \\ ${ }^{6}$ INRA, UMR1300 Bioagression, Epidémiologie et Analyse de risque, Atlanpole La Chantrerie, \\ F-44307 Nantes, France \\ ${ }^{7}$ ONIRIS, Bioagression, Epidémiologie et Analyse de Risque, Atlanpole La Chantrerie, F-44307 Nantes, France \\ 8 INRA,UMR1225 IHAP Interactions Hôtes-Agents Pathogènes, F-31076 Toulouse, France \\ ${ }^{9}$ ENVT, IHAP Interactions Hôtes-Agents Pathogènes, F-31076 Toulouse, France \\ 10 UR1282 IASP Infectiologie Animale et Santé Publique, F-37380 Nouzilly, France \\ 11 INRA,UE1277 PFIE Plate-Forme d'Infectiologie Expérimentale, F-37380 Nouzilly, France \\ 12 INRA, UMR1323 RiTME, 65 Boulevard de Brandebourg, F-94205 Ivry-sur-Seine, France \\ 13 INRA, USC Bartonella et Tiques, ANSES, F-94700 Maisons-Alfort, France \\ 14 INRA, UMR1161 Virologie, 7 avenue du Général de Gaulle, F-94704 Maisons-Alfort, France \\ ${ }^{15}$ ENVA, ANSES, Virologie, 7 avenue du Général de Gaulle, F-94704 Maisons-Alfort, France \\ 16 INRA, Département de Santé Animale, F-37380 Nouzilly, France \\ Courriel :Christian.Ducrot@clermont.inra.fr
}

Au fil des crises sanitaires, les attentes des pouvoirs publics ont évolué vis-à-vis de la recherche en santé animale. L'approche One world, one health «un monde, une santé» met l'accent sur les interconnections étroites entre santé humaine et animale, et sur la globalisation des problèmes de santé à l'échelle de la planète. Dans ce contexte, une réflexion a été conduite sur les enjeux de la santé animale et les spécificités de la recherche dans ce domaine.

Les représentations d'une recherche en santé animale et les attentes qu'en ont les financeurs et les organismes de recherche ont considérablement évolué. Un nombre croissant de parties prenantes considère cette recherche sous l'angle restrictif des dangers et des risques que les maladies animales traditionnelles ou émergentes font directement courir à la santé de l'Homme. De plus, certains considèrent la santé comme un capital commun à l'ensemble des espèces, à la fois humaine et animales, qui serait garanti par une médecine unique irriguée par la recherche médicale. Dans ce contexte évolutif, une réflexion sur les spécificités de la recherche en santé animale, ses enjeux et les enrichissements particuliers qu'elle prodigue à la recherche générique du champ de la santé est apparue nécessaire. Ce texte résume les résultats de cette réflexion ${ }^{1}$. Elle intègre les enjeux à l'échelle mondiale et dépasse donc le strict cadre national. Elle est présentée en trois parties. La première aborde les enjeux de la santé animale et de la recherche en santé animale, dont la plupart ne relèvent pas de maladies zoonotiques. La deuxième partie décrit les spécificités de la recherche en santé animale, liées aux contraintes scientifiques, mais aussi à leur enra- cinement dans une réalité agricole et économique, et aux points de vue à partir desquels sont posées les questions scientifiques. La troisième partie présente une réflexion sur l'organisation de la recherche en santé animale. La conclusion propose des évolutions permettant de garantir une recherche adaptée aux spécificités du domaine tout en favorisant les partenariats avec la recherche en santé humaine. Cette réflexion a été volontairement limitée aux animaux de rente; les animaux de compagnie et la faune sauvage ne sont évoqués que de manière comparative.

\footnotetext{
${ }^{1}$ Cette réflexion a été menée par un groupe de travail pluridisciplinaire d'une quinzaine de chercheurs issus d'unités de recherche de l'INRA, des écoles vétérinaires et de l'AFSSA (L'AFSSA et l'AFSSET ont fusionné le 1er juillet 2010 pour devenir l'ANSES (Agence nationale de sécurité sanitaire de l'alimentation, de l'environnement et du travail www.anses.fr), associant microbiologistes, pathologistes, infectiologues, épidémiologistes, généticien, zootechnicien, économiste et sociologue. Outre sa propre réflexion, ce groupe s'est appuyé sur des références bibliographiques et a consulté des responsables de l'Organisation mondiale de la santé animale (OIE), un chercheur de l'Institut Pasteur, et des cadres d'une société pharmaceutique productrice de vaccins (Merial). Son travail a ensuite été revu par un cercle de chercheurs seniors de l'INRA, d'universités française et belge, et d'une école vétérinaire.
} 


\section{1 / Enjeux des recherches en santé animale}

\section{1 / Santé animale et santé publique vétérinaire}

Les maladies animales peuvent être schématiquement réparties en trois catégories. Les maladies épidémiques majeures sont caractérisées par une forte contagiosité et des impacts considérables sur les cheptels (par exemple fièvre aphteuse, pestes porcines, Influenza aviaire à virus hautement pathogène); l'enjeu est de les éradiquer d'un territoire quand c'est possible, et leur survenue dans une population totalement sensible peut avoir des conséquences sanitaires et économiques très importantes. D'autres maladies infectieuses transmissibles présentent une contagiosité ou des impacts moindres, et sont plus souvent présentes de façon endémique dans les populations. Les maladies multifactorielles sont quant à elles provoquées par un ensemble de facteurs de risque liés notamment à la conduite d'élevage, avec parfois participation d'agents pathogènes très répandus en élevage ; appelées «maladies de production», elles sont présentes dans la grande majorité des exploitations d'élevage, à des fréquences très variables. Parmi les maladies transmissibles, les maladies zoonotiques le sont également à l'Homme. De plus, les animaux peuvent être porteurs sains d'agents pathogènes pour l'Homme, qui n'ont pas ou peu d'effet sur la santé de l'animal luimême (par exemple Salmonelles et Campylobacter).

Reprenant un cadre produit par des instances internationales (OMS, FAO, OIE) $)^{2}$ (WHO 2002), l'Académie Vétérinaire de France (séance du 15 mai 1997) a défini la santé publique vétérinaire comme «l'ensemble des actions qui sont en rapport direct ou indirect avec les animaux, leurs produits et sous-produits, dès lors qu'elles contribuent à la protection, à la conservation et à l'amélioration de la santé de l'Homme, c'est à dire son bien-être, physique, moral et social». La santé publique vétérinaire couvre les champs d'intervention relatifs à la santé et à la protection des animaux, la sécurité sanitaire des aliments et la préservation de l'environnement Entrent ainsi dans le domaine d'activité de la santé publique vétérinaire le contrôle des maladies animales ayant un impact direct sur la santé de l'Homme de par leur caractère zoonotique, mais aussi le contrôle de l'ensemble des maladies animales non transmissibles à l'Homme mais dont la présence est de nature à entraîner des pertes de production importantes et des perturbations des marchés.

\section{2 / Enjeux de la santé animale}

Les enjeux liés à la maîtrise de la santé animale sont de quatre ordres :

- Des enjeux économiques pour un ensemble de maladies qui ont un impact sur la viabilité économique des exploitations d'élevage (notamment les maladies d'élevage ou maladies endémiques qui entraînent des pertes de production, des coûts de prévention ou de traitement, des désorganisations de l'élevage et du travail de l'éleveur ${ }^{5}$ ), et des filières de production animales (notamment les maladies épidémiques, en relation avec leur effet sur la production, les impacts des réglementations sanitaires sur le marché, et les freins au commerce). Dans les pays du Nord, ces maladies pèsent lourdement sur la compétitivité économique des élevages, des entreprises, et des filières de productions animales dans leur ensemble. Dans les pays du Sud, s'ajoutent à ces conséquences des risques de pénurie alimentaire, de décapitalisation (dans la mesure où le cheptel constitue un capital sur pied, seule épargne et seule assu-

\section{Rôle des services vétérinaires}

Dans un éditorial du 15 novembre 2009 sur la législation vétérinaire comme fondement de toute politique de santé animale efficace ${ }^{3}$, Bernard Vallat, Directeur général de I'OIE ${ }^{4}$ rappelle que "les Services vétérinaires nationaux ont un rôle déterminant à jouer, non seulement pour prévenir et contrôler les maladies animales mais aussi pour améliorer la sécurité alimentaire, la nutrition, la sécurité sanitaire des aliments, la santé publique vétérinaire et l'accès aux marchés des animaux et de leurs produits au niveau mondial». L'accent est mis tout autant sur la santé de l'Homme et du consommateur que sur la sécurité alimentaire (sécurité de l'approvisionnement en produits alimentaires) et le fonctionnement des marchés d'animaux et produits d'origine animale. rance sociale d'une grande partie de la population), de perte de la force motrice des animaux de trait et de labour (provoquant une diminution de l'efficience agricole en général).

- Des enjeux de santé publique qui concernent trois domaines : les zoonoses, maladies infectieuses ou parasitaires transmissibles des animaux à l'Homme, que ce soit par contagion (par exemple tuberculose, brucellose, certains virus Influenza), via des vecteurs (maladie de West Nile, fièvre de la vallée du Rift, maladie de Lyme) ou par l'alimentation (ESB, toxi-infections alimentaires); la résistance aux antibiotiques (antibiorésistance) ; et les résidus médicamenteux dans les produits animaux.

- Des enjeux environnementaux relatifs à l'impact de l'agriculture, qui concernent le rejet des xénobiotiques dans l'environnement (résidus médicamenteux), la diffusion de l'antibiorésistance, et les maladies infectieuses transmissibles entre animaux domestiques et faune sauvage (à l'instar de la tuberculose bovine détectée dans la faune sauvage).

- Des enjeux de bien-être animal, qui vont de pair avec les évolutions réglementaires dans ce domaine. Les maladies entraînent souffrance et douleurs, et leur absence est un des critères retenus dans la grille d'évaluation du bien-être animal issue du projet Welfare quality (Veissier et al 2010).

Dans un rapport récent sur la situation mondiale de l'alimentation et de l'agriculture consacré à l'élevage (FAO 2009), la $\mathrm{FAO}^{6}$ résume ces différents enjeux : «Parce qu'elles peuvent mettre en danger la santé humaine, perturber les marchés et le commerce, réduire la productivité et aggraver la pauvreté, les maladies animales ainsi que les maladies d'origine alimentaire causées par le manque d'hygiène alimentaire, posent un problème à tout un chacun. L'amélioration de la gestion de l'élevage, en vue de prévenir et de maîtriser ces maladies peut être porteuse de bienfaits économiques, sociaux et sanitaires pour les pauvres et pour l'ensemble de la société». Parmi les quatre messages clés de ce rapport sur l'élevage dans le monde, il est relevé que «les maladies animales présentent des risques systémiques auxquels il importe de remédier»».

\footnotetext{
${ }^{2}$ L'OMS définit actuellement la santé publique vétérinaire comme «the sum of all contributions to the physical, mental and social well-being of humans through an understanding and application of veterinary science» (WHO, 2002) (Consulté le 2010/11/17).

$3 \mathrm{http}: / /$ www.oie.int/fr/edito/fr edito.htm?e1d3 consulté le $02 / 02 / 2010$

4 Organisation mondiale de la santé animale.

5 Entre dans ce cadre l'enjeu de participer à l'étude de systèmes innovants intégrant la santé dans les objectifs et facteurs de production et recherchant un optimum de production intégrant les aspects santé et environnement.

6 Organisation des Nations Unies pour l'Alimentation et l'Agriculture.
} 
Santé animale - particularités des animaux de production, de compagnie, et de la faune sauvage

Pour les animaux de production, les maladies infectieuses et parasitaires sont très largement majoritaires, même si les troubles métaboliques et dégénératifs existent évidemment, le plus souvent en relation avec une maîtrise insuffisante des systèmes de production. L'animal de compagnie ou de sport présente par contre un profil pathologique qui se rapproche singulièrement de celui de l'Homme (troubles endocriniens, cancers, maladies dégénératives nerveuses ou ostéo-articulaires, voire obésité, vieillissement), d'où une moins grande présence de la pathologie infectieuse et parasitaire au profit de la médecine interne, cancérologie, ou endocrinologie, bien que les médicaments antibiotiques et antiparasitaires ainsi que les vaccins représentent environ $75 \%$ de la consommation des médicaments chez les animaux de compagnie. Enfin, la faune sauvage non captive constitue un objet plus récent d'investigation en santé animale, consacré aux réservoirs épidémiologiques majeurs d'agents potentiellement zoonotiques (par exemple lyssavirus des chauves-souris, virus Influenza aviaires) ou aux sentinelles de la contamination et de la pollution toxicologiques de l'environnement.

Pour l'ensemble de ces maladies, les enjeux concernent au premier chef les exploitations agricoles, mais aussi les filières économiques qui en dépendent : animaux vivants, produits d'origine animale, intrants de l'agriculture et services. Au-delà, sont aussi concernés les consommateurs et les citoyens, tant en termes de sécurité alimentaire quantitative et qualitative, que de santé publique. L'élevage et les filières agroalimentaires occupent une place centrale dans les pays industrialisés ${ }^{7}$ (sécurité alimentaire, irrigation d'une activité économique considérable liée à l'approvisionnement de l'élevage, y compris l'industrie pharmaceutique, et à la valorisation et au commerce des produits agricoles et alimentaires souvent technologiquement très élaborés), comme dans les pays en développement (agriculture de subsistance, sécurité alimentaire, apport de protéines de qualité). Dès lors, les enjeux économiques de la santé animale, sans même mentionner les risques de bioterrorisme, représentent des enjeux stratégiques incontournables, même s'ils demeurent moins médiatisés que les enjeux de santé publique.

De plus, ces différents enjeux ne sont pas indépendants les uns des autres. Par exemple, le risque de présence de résidus médicamenteux dans les produits animaux, de même que le risque d'antibiorésistance provenant du monde animal, tous deux enjeux de santé publique, sont très directement corrélés à la fréquence des maladies enzootiques qui impactent l'équilibre économique des filières de productions animales, et donc relèvent avant tout des enjeux économiques liés à la maîtrise de la santé animale.

\section{3 / Importance des maladies, hiérarchisation des enjeux}

La hiérarchisation des différents enjeux liés aux maladies animales et à leur maîtrise est difficile.

Il y a plusieurs façons d'évaluer l'importance des maladies animales. La première est d'estimer leurs impacts sur les performances zootechniques et économiques. Les taux de mortalité des animaux dans les systèmes d'élevage d'Europe de l'Ouest ont des valeurs moyennes non négligeables dans certains groupes d'âge, et peuvent atteindre des valeurs élevées dans les troupeaux où la pathologie est mal maîtrisée ; à titre d'exemple, la mortalité des veaux avant sevrage est en moyenne de $12 \%$, celle des vaches laitières de $3 \%$, celle des porcelets avant sevrage de $20 \%$ (en incluant les mort-nés), pour encore environ $7 \%$ de pertes et saisies entre le sevrage et l'abattage des porcs charcutiers. A la mortalité s'ajoutent divers coûts de maîtrise des maladies. Aussi, en moyenne, l'impact économique des troubles de santé courants dans des exploitations laitières de dimension moyenne (44 vaches laitières, Pays de la Loire) a été estimé en 2000 à environ $10000 €$ (Fourichon 2001), et l'impact économique des troubles respiratoires des veaux non sevrés dans des exploitations bovines allaitantes en système naisseurengraisseur représentait en 2000 en moyenne $12 \%$ du revenu de l'éleveur (Assié et al 2001). L'impact économique actuel des seules infections intramammaires des vaches laitières en France peut être évalué à 350 millions $€ /$ an, principalement à cause de réductions de productivité et de longévité, de réduction des prix de vente du lait et des coûts des mesures de prévention et de traitement. En aviculture, les coccidioses ont un impact majeur; sur la base d'un modèle britannique (Williams 1999), leur impact économique mondial est estimé à plus de deux milliards de dollars, principalement en raison de leur impact sur la production et l'efficacité alimentaire.

La liste des maladies notifiables à l'OIE comprend les maladies infectieuses transmissibles jugées, au niveau international, les plus préjudiciables d'un point de vue économique et santé publique $^{8}$; parmi les 119 maladies répertoriées, seulement 31 sont zoonotiques à un degré ou un autre (Ganière 2004). Par ailleurs, les priorités affichées par les instances internationales (OMS, FAO, OIE), fédérées dans le programme GLEWS ${ }^{9}$ (Global Early Warning and Response System for Major Animal Diseases, including Zoonoses) en termes de surveillance et monitoring des maladies animales, découlent d'une première approche initiée par l'OMS privilégiant des maladies zoonotiques. C'est pourquoi la liste de GLEWS comprend 6 maladies non zoonotiques et 19 zoonotiques.

Sur la base de la production de vaccins, il faut souligner que près de $100 \%$ des vaccins commercialisés en santé animale protègent vis-à-vis d'agents pathogènes strictement animaux. Le vaccin antirabique est un des rares vaccins vétérinaires destinés à protéger l'Homme. Certains autres vaccins vétérinaires, tels que celui contre la leptospirose, visent un agent zoonotique mais sont utilisés pour protéger les animaux de compagnie ; ils n'ont pas vocation à protéger les animaux au titre de la santé publique.

Un dernier éclairage est fourni par l'étude bibliométrique 2006-2009 menée dans le cadre du programme européen Era-Net EMIDA (Emerging Infectious Diseases of Animals) (de Rycke 2009), focalisé sur les maladies infectieuses et parasitaires des animaux de production. La cartographie réalisée montre que la santé animale est située à l'intersection d'autres champs disciplinaires comme la santé humaine, mais aussi la santé de la faune sauvage et des écosystèmes, la nutrition animale, la génétique animale ou encore le bien-être animal. L'étude montre également que $20 \%$ des 12000 publications recensées sur les maladies infectieuses traitent des

\footnotetext{
${ }^{7}$ A titre d'illustration, le cheptel français compte environ 20 millions de bovins, 9 millions de petits ruminants, 15 millions de porcs, 247 millions de volailles (Données 2007). Dans notre pays, la production de lait et de viande constitue plus de $30 \%$ de la valeur de la production agricole française (environ 24 milliards d'euros en 2007) et les filières animales font vivre plus de 500000 actifs, toutes professions confondues (Bimagri hors-série $\mathrm{n}^{\circ} 22$, janvier 2009 ).

8 http://www.oie.int/eng/maladies/en classification2010.htm, consulté le 30 avril 2010.

9 Global Early Warning and Response System; http://www.glews.net/ consulté le 12/04/2010.
} 
Panorama de la recherche sur les maladies infectieuses et parasitaires en santé animale

Dans le cadre du projet européen Star-Idaz, les travaux de recherche menés au niveau international sur les maladies infectieuses et parasitaires des animaux de rente ont été analysés (de Rycke 2010) à partir de l'étude des 28750 publications scientifiques internationales parues sur le sujet de 2006 à juin 2010. Cette étude illustre le maillage scientifique dense existant entre les pays importants en matière de recherche, dont fait partie la France. Elle montre par ailleurs le poids relatif des différentes espèces de rente (figure 1) et des agents pathogènes étudiés (figure 2) dans les travaux de recherche conduits au cours des dix années passées (compte tenu du délai entre mise en œuvre des travaux et publication).

Par ordre d'importance décroissante, les travaux à l'échelle mondiale portent sur ruminants pour moitié, puis volailles et porc pour un tiers, le reste réparti entre les autres espèces. La situation en France est assez semblable avec quelques nuances. Concernant les agents pathogènes, trois quarts des recherches internationales portent sur bactéries et virus, le quart restant étant réparti entre protozoaires, helminthes, ectoparasites et prions. La situation française ressemble à la moyenne mondiale, avec toutefois un développement relatif plus important des travaux sur les prions.

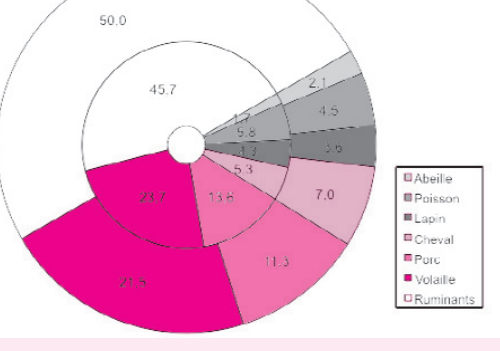

Monde : cercle extérieur

France : cercle intérieur

Figure 1. Répartition des travaux scientifiques sur les maladies infectieuses et parasitaires en fonction du type d'espèce de rente concernée (dans le monde et en France).

Monde : cercle extérieur

France : cercle intérieur

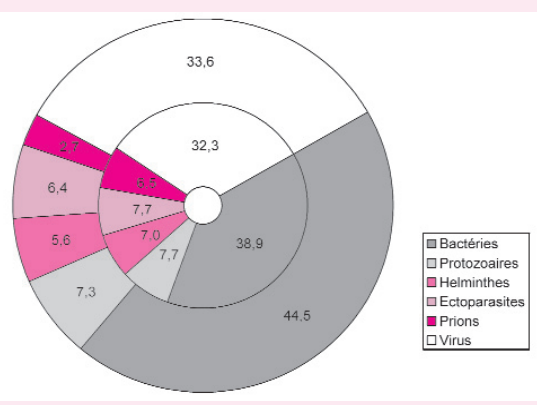

Figure 2. Répartition des travaux scientifiques sur les maladies infectieuses et parasitaires en fonction du type d'agent pathogène étudié (dans le monde et en France).

zoonoses et de la sécurité sanitaire des aliments, et sont donc directement liées à des enjeux de santé publique. Ce constat signifie a contrario que $\mathbf{8 0 \%}$ des publications traitent de maladies exclusivement animales, dont les enjeux sont d'abord économiques, environnementaux, ou de bien-être animal.

\section{4 / Recherche en santé animale}

Face à l'ensemble de ces enjeux, audelà des connaissances scientifiques déjà acquises, il existe des besoins de recherche nombreux en santé animale. Ainsi, à titre d'exemple, le schéma stratégique du Département de Santé Animale de l'INRA identifie des questions dans trois grands domaines: la connaissance des agents pathogènes, la relation entre l'hôte (animal infecté) et l'agent pathogène, l'interaction des pathogènes et des hôtes dans les populations animales. Les travaux à mettre en œuvre ont donc pour but de proposer des outils de maîtrise de l'exposition des animaux domestiques aux agents pathogènes, renforcer la résistance des hôtes aux agents pathogènes (par la vaccination notamment), ou traiter les animaux malades. La maîtrise et le contrôle des maladies par des actions de lutte et de prévention requièrent aussi des travaux d'évaluation de l'impact économique et social des plans de gestion sanitaire.

A côté de ces enjeux finalisés, il existe des enjeux plus fondamentaux de production de connaissances génériques sur les modèles animaux. Les travaux menés dans ce domaine nourrissent la connaissance en biologie grâce à la biologie comparée. La diversité des espèces modèles étudiées, la disponibilité de dispositifs expérimentaux et de matériel biologique, ainsi que la maîtrise de modèles infectieux particuliers sont des atouts importants pour ces travaux qui produisent des connaissances sur le vivant, sans forcément d'application immédiate, mais qui peuvent ultérieurement se montrer très utiles (exemple de molécules - défensines, récepteurs Toll - de l'immunité innée identifiées chez les invertébrés, ayant des applications vaccinales ou immunomodulatrices chez l'Homme et l'animal domestique).

\section{2 / Spécificités des recher- ches en santé animale}

La santé animale présente des spécificités en matière d'objectifs, méthodes, modèles biologiques et questionnements scientifiques. En revanche, il existe également des domaines de convergence générique et méthodologique avec la santé humaine.

\section{1 / Spécificités d'objectifs, de méthodes et de modèles biolo- giques}

En premier lieu, l'élevage des espèces animales de production (food animals pour les Anglo-Saxons) constitue une entreprise économique dont la finalité est de dégager des revenus. Dans ce contexte, la santé animale est un des facteurs que l'éleveur doit piloter, en faisant en sorte de minimiser les risques sanitaires auxquels son troupeau est exposé et de contenir à moindre coût l'impact des maladies. Dans un système d'élevage donné et pour un niveau de production donné, les maladies sont par ailleurs étroitement liées au mode de conduite de l'élevage, et notamment à des paramètres relatifs à la qualité du logement, de la nutrition, et de l'hygiène. Le processus d'intensification de l'élevage qu'a connu le monde agricole depuis une cinquantaine d'années a accentué ces tensions entre limitation des intrants, augmentation de la production et risque de maladies.

Il s'ensuit la nécessité d'une médecine de population dans les élevages, mais aussi celle d'une recherche sur les maladies qui tienne compte de manière spécifique de ces connexions étroites entre santé et zootechnie au sens large. Ce constat implique de collaborer de manière approfondie d'une part avec les autres disciplines des sciences animales, et d'autre part avec les différents acteurs du monde de l'élevage. Seule une 


\section{Spécificités liées à l'approche des maladies en élevage}

Que ce soit pour les maladies transmissibles dues à des agents pathogènes variés (mammites, diarrhées néonatales, maladies respiratoires des jeunes par exemple), les maladies parasitaires, les maladies métaboliques, et dans une certaine mesure pour les maladies infectieuses transmissibles, la présence de ces maladies dans les troupeaux, leur importance et plus encore leur maîtrise, sont en grande partie dépendantes de paramètres relatifs à la conduite des élevages. Ainsi par exemple, certaines maladies dites "d'élevage», d'origine multifactorielle, sont en partie la résultante d'une maîtrise insuffisante des risques liés aux pratiques d'élevage (par exemple hygiène du logement, densité animale, contacts entre animaux, équilibre des rations alimentaires...).

Dans ce contexte, l'étude des maladies animales en élevage, qui relève de l'épidémiologie animale et de l'écopathologie (Ganière et al 1991) développée dans les années soixante-dix, tient son originalité dans le fait de considérer la maladie comme une "sortie» du système d'élevage piloté par l'éleveur. Ceci oblige à prendre en considération dans l'étude des facteurs agissant sur les maladies nombre de paramètres relatifs à la description du système, et à faire en sorte de différencier lors de l'analyse les facteurs de risque des facteurs de confusion et autres indicateurs de risque. Aussi, la connaissance intime du milieu de l'élevage est-elle une nécessité pour étudier les maladies en élevage.

Au fil du temps, le questionnement en matière de santé animale en élevage a dépassé le seul objectif de rentabilité économique par la réduction de la fréquence des maladies, pour aborder aussi la qualité sanitaire des produits d'origine animale, la réduction de l'usage des xénobiotiques, et le bien-être animal, dans un souci de santé publique et de développement durable. Cet enjeu multiple amène à réfléchir au meilleur équilibre possible entre ces différents paramètres. Avancer dans cette démarche implique pour les acteurs de la santé animale, que ce soit du point de vue recherche ou développement, une connexion étroite avec les sciences de l'élevage et la profession agricole. recherche en étroite relation avec les pratiques réelles des éleveurs et des illustratif, une recherche intégrée sur la gestion de la santé en élevage, dans le cadre de la mise en œuvre d'une agriculture raisonnée, suppose une bonne connaissance du monde de l'élevage, impose une collaboration étroite entre disciplines des productions animales, génétique, économie de l'élevage, sociologie et santé animale, et repose sur un partenariat avec les acteurs de la santé animale en élevage.

Une deuxième particularité de la recherche en santé animale est la très grande prédominance des affections infectieuses ou parasitaires, au moins pour les animaux de production, avec une très grande diversité d'entités pathologiques, et un répertoire très large d'agents pathogènes impliqués. A ce titre, les équipes conduisant des recherches en santé animale doivent nécessairement s'intéresser à une grande variété de familles d'agents pathogènes, et constituent un vivier de compétences rares et précieuses, en virologie, bactémédicales.

Une troisième spécificité de la recherche en santé animale est liée aux particularités génétiques des animaux d'élevage. La durée d'évolution naturelle des espèces animales, qui aboutit à la diversité des espèces, est très supérieure aux phases de domestication, qui aboutissent à la diversité des races, et à la période de sélection intensive conduite depuis une cinquantaine d'années, avec une amélioration considérable des profilières animales est pertinente. A titre riologie, parasitologie et entomologie ductions mais une forte homogénéisation génétique des animaux d'élevage. Une particularité de l'élevage est en effet la possibilité d'une action, diri- gée par l'Homme, de sélection des animaux sur des caractères génétiques particuliers, de production le plus souvent (par exemple la quantité de lait) mais également de résistance aux maladies (par exemple contre la tremblante du mouton). La compréhension des bases génétiques de la sensibilité aux maladies infectieuses nécessite donc de prendre en compte la durée de coévolution, la diversité génétique et la dynamique évolutive respective des hôtes et des pathogènes. L'amélioration génétique de la réponse immune est un objectif de sélection complexe. Elle peut être dirigée contre un objectif unique (un pathogène) mais fluctuant (en raison de sa dynamique évolutive rapide); elle peut aussi viser une meilleure immunocompétence globale, mais avec généralement une corrélation négative avec la sélection sur les caractères de production.

Les espèces animales domestiques sont relativement variées, et très éloignées les unes des autres d'un point de vue phylogénétique, ainsi que de la souris. Elles ont de ce fait des spécificités physiologiques, concernant entre autres le métabolisme, le système immunitaire, la placentation, la physiologie

\section{Spécificités de la recherche en santé animale liées aux espèces}

L'espèce de référence des études en santé humaine (infectiologie, cardio-vasculaire, cancer, physiologie) est la souris. L'étude des mécanismes moléculaires passe en général d'abord par cette espèce de mammifères puisqu'elle est la seule où l'invalidation spécifique de gènes peut être conduite efficacement et elle permet des investigations sur de grands nombres d'animaux. Les connaissances établies chez la souris sont ensuite transposées aux espèces d'application : l'Homme et les animaux domestiques. Or cette transposition n'est souvent pas satisfaisante. En effet, il est décevant de constater l'inefficacité des traitements anti-cancéreux ou l'inefficacité des vaccins chez l'Homme et les espèces domestiques alors que les résultats utilisant les mêmes stratégies étaient excellents chez la souris. On peut par exemple mentionner la vaccination génétique, efficace chez la souris, et qui fonctionne mal chez l'Homme et les ruminants, ou le défaut de transposition pour la vaccination par voie muqueuse avec des antigènes inertes.

Ces différences entre espèces tiennent à la physiologie spécifique de chaque espèce et à son interaction particulière avec un agent pathogène donné. L'Homme et la souris appartiennent au superordre des Supra-primates; en revanche, les espèces animales domestiques incluent des espèces phylogénétiquement très éloignées de la souris et de l'Homme, tels les poissons, les oiseaux et les mammifères Laurasiatheria (vache, porc, cheval, carnivores). Ces espèces se distinguent phylogénétiquement de l'Homme et de la souris et ont des spécificités de physiologie et d'environnement qui conditionnent les dispositifs biologiques de maîtrise de leur santé. Ainsi les poissons ont des spécificités propres à leur milieu particulier de vie, à leur biologie cellulaire (facilité de transfert de gènes, efficacité de la vaccination par ADN), à leur système immunitaire plus primitif (spécificité de la mémoire immune, répertoire lymphocytaire différent, interaction avec les agents pathogènes par les nageoires). Les ruminants sont polygastriques et ont des spécificités nutritionnelles propres. Ils ont une placentation différente de celle de l'Homme et de la souris et une physiologie mammaire particulière. Ces spécificités ont un impact sur le transfert de l'immunité au jeune et sur les méthodes vaccinales de protection néonatale. Les artiodactyles (porc/ruminants) présentent un développement immunitaire particulier (développement néonatal immun dans les plaques de Peyer iléales, réponse des cellules dendritiques à des signaux différents de la souris, répertoire immunitaire différent de I'Homme et de la souris).

Ainsi, pour répondre à la demande sociétale, une recherche spécifique en physiologie/immunologie/infectiologie chez l'Homme et chez l'animal domestique au sens large se doit d'exister. Par exemple, le développement de stratégies vaccinales nouvelles doit impérativement être réalisé dans l'espèce cible, dont le système immunitaire est stimulé par des signaux spécifiques liés à l'expression de récepteurs particuliers, qui se traduit par le fait que les espèces domestiques répondent de manière différente de la souris aux vecteurs vaccinaux recombinants. 
Les modèles animaux domestiques pour la recherche à visée humaine

La souris se révèle souvent être un modèle inadéquat d'études physiopathologiques, prophylactiques ou thérapeutiques pour l'Homme. Ceci tient à la taille réduite de l'espèce, à des considérations physiologiques, à l'absence de pathologie naturelle correspondante. Concernant ce dernier point, il est souvent nécessaire d'infecter la souris avec l'agent pathogène humain, et créer ainsi un modèle artificiel sans symptômes pertinents. Aussi, les espèces domestiques sont-elles dans certaines circonstances de bien meilleurs modèles d'étude pour la recherche à visée humaine.

Les espèces domestiques peuvent être infectées par des virus ayant coévolué avec leu hôte. Ces maladies présentent des similitudes de mécanismes moléculaires et physiopathologiques avec les affections humaines, sans pour autant être zoonotiques. Ainsi le porc, infecté par un virus grippal qui s'est adapté au porc, souffre d'un syndrome grippa proche de celui rencontré chez l'Homme infecté par un virus grippal humain. Le jeune veau est infecté par un virus respiratoire syncytial distinct de l'humain, et développe une pathologie broncho-pulmonaire proche de celle de l'enfant. Ces affections animales permettent ainsi de développer des stratégies thérapeutiques, vaccinales et diagnostiques adaptables ou extrapolables à l'Homme.

D'autre part, par convergence évolutive, certaines espèces domestiques présentent des similitudes fonctionnelles avec l'Homme, plus que la souris : par exemple le porc pour la structure de la peau (étude de thérapie ou vaccination par voie transcutanée), le mouton pour la pathologie respiratoire (étude immunologique du traitement de l'asthme), le porc pour les maladies cardio-vasculaires, le développement de mélanomes spontanés dont la progression tumorale est semblable à celle observée chez l'Homme.

Enfin, les animaux domestiques, du fait de leur grande taille, permettent également d'étudier de manière originale, non faisable chez la souris, des fonctionnements immuns. Ainsi, il est possible de cathétériser les vaisseaux lymphatiques chez le porc, la vache et le mouton, pour étudier directement dans la lymphe des populations de leukocytes migrant à l'état basal, lors d'infections, ou de vaccination, permettant de suivre en temps réel certains aspects de la réponse immune.

Pour ces différentes raisons, les connaissances approfondies en physiopathologie des animaux domestiques et l'existence de plates-formes performantes d'expérimentation animale sont très utiles en recherche biomédicale. D'une manière générale, la diversité des modèles (espèces animales) étudiés, base de la biologie comparée, est importante pour produire des connaissances générales, qui peuvent avoir des applications diverses, notamment en biologie humaine.

mammaire, qui induisent des relations hôtes-pathogènes spécifiques et des approches vaccinales différentes. La recherche et l'expérimentation sur les espèces «cibles» (le poisson, la poule, le porc, les ruminants) est de fait souvent une nécessité, mais aussi un atout car les résultats de recherche sont directement exploitables sur l'espèce, sans nécessité de valider l'extrapolation faite à partir d'un modèle animal, contrairement aux travaux menés pour une application en santé humaine.

Enfin, il existe des particularités liées aux types de mesures de lutte contre les maladies animales, ou gestion sanitaire. Au-delà de la vaccination ou de la protection des élevages, la police sanitaire visant les maladies contagieuses listées par le Code Rural comprend une panoplie de méthodes de lutte, y compris parfois des mesures d'abattage des animaux pour éliminer ceux qui sont à risque pour les animaux indemnes ou pour l'Homme. Ces pratiques induisent de fait des questions de recherche spécifiques sur les dispositifs d'intervention. Au premier rang de ces questions, il faut noter le besoin de mise au point d'outils sérologiques permettant de distinguer les animaux vaccinés des animaux infectés, car les mesures de maîtrise des maladies sont ne semble pas qu'elles aient un impact notable sur la façon de conduire la recherche en santé animale.

\section{2 / Spécificités de questionne- ment scientifique}

A côté des aspects développés précédemment, une des principales spécificités des recherches en santé animale relève des questions scientifiques posées, qui le sont du point de vue de la santé de l'animal et non de celle de l'Homme. Ainsi, même dans le cas des agents zoonotiques, les questions posées par les équipes de santé animale ne sont pas les mêmes que celles posées par les équipes biomédicales. A titre d'exemple, dans le cas d'agents vectoriels zoonotiques, par exemple les Borrelia agents de la maladie de Lyme, l'accent sera mis sur le rôle des animaux comme réservoirs d'agents potentiellement pathogènes pour l'Homme, et sur les composantes qui permettent le développement d'un agent infectieux dans son hôte réservoir versus l'Homme. Pour les maladies à prions, un positionnement en santé animale conduira à étudier la diversité de souches rencontrées chez l'animal, à essayer de décrypter les interactions entre la souche infectieuse et l'espèce hôte. Plus généralement, les études sur les interactions hôte/agent pathogène sous l'angle de la santé animale se sont souvent révélées fructueuses aux plans fondamental et appliqué du fait en particulier des connaissances de la génétique de l'hôte infecté et de la possibilité de mettre en place des protocoles avec une cohorte expérimentale à statut génétique défini. C'est par exemple le cas de la mise en évidence chez le mouton de la modulation de la susceptibilité à la tremblante en relation avec le polymorphisme du gène codant pour la protéine prion.
Il existe bien entendu d'autres particularités du monde de l'élevage, mais il différentes pour ces catégories d'animaux, et l'ensemble des questions linterêt économique com etions de leur appropric par les éleveurs et les autorité publiques.

\section{Communauté de recherche en santé animale et production scientifique}

Les enjeux de santé animale présentés dans ce texte se traduisent en questions de recherche finalisées. Aussi le positionnement des recherches dans ce domaine est-il stratégique, au sein de la communauté scientifique de santé animale. Dans le même temps, il doit aussi laisser la place, l'initiative et la légitimité pour des développements de recherche plus fondamentaux sur les modèles animaux, qui représentent un atout dans certains domaines scientifiques et contribuent à la production de connaissances issues de la biologie comparée. Le positionnement individuel de chaque chercheur n'est pas aisé entre ces deux orientations de la recherche : plus finalisée ou plus fondamentale. Aussi cette complémentarité peut-elle être recherchée à l'échelle des équipes ou des unités de recherche.

Le choix des revues dans lesquelles le chercheur souhaite publier rejoint celui de la définition de la communauté scientifique dans laquelle il se situe. Il est important que les équipes qui publient dans le champ disciplinaire de la santé animale (Veterinary Sciences) soient pleinement reconnues, puisque ce champ correspond aux enjeux finalisés de leurs recherches. II est néanmoins important que soient reconnues aussi les aspirations des équipes développant une recherche plus fondamentale, qui doivent prétendre publier dans les revues de leur communauté disciplinaire (immunologie, bactériologie...).

Ce double positionnement au sein des équipes de recherche en santé animale est légitime, mais il est susceptible de créer des tensions et des incompréhensions si la politique scientifique et la politique qualité de la production scientifique qui l'accompagnent ne mentionnent pas explicitement ces deux facettes de la recherche en santé animale. 
Il apparaît donc que, bien que travaillant sur les mêmes agents et avec les mêmes outils, les questionnements en santé animale peuvent être différents de ceux en biologie humaine et conduisent à produire une connaissance complémentaire. Il s'ensuit des possibilités de collaborations entre équipes en santé animale et biologie humaine, chacun ayant en raison des questions qu'il se pose et ses réseaux de partenariat «naturels» (hôpitaux versus exploitations agricoles ou environnement), accès à des types de prélèvements différents et complémentaires. Par exemple, une collaboration pourra porter sur la comparaison, à visée épidémiologique, de souches de Bartonelles prélevées chez l'Homme et différentes espèces animales.

\section{3 / Domaines de convergence générique et méthodologique avec la santé humaine}

Dans certains domaines, les recherches menées en biologie humaine et en santé animale mobilisent des outils similaires, voire les mêmes modèles pour répondre aux questions de recherche. Dans ce cas, notamment dans le cadre de l'étude des agents pathogènes zoonotiques, la seule différence tient au questionnement.

Dans certains cas néanmoins, les équipes en biologie humaine et en santé animale abordent les mêmes questions, et il n'y a plus alors de spécificité évidente à la santé animale. L'évolution de projets de recherche centrés au départ sur la santé animale a pu conduire progressivement les équipes concernées à se poser des questions de plus en plus pointues sur des modèles identiques à ceux de la biologie humaine. Citons à titre d'illustration des approches fondamentales sur les mécanismes moléculaires d'invasion des cellules cibles par le virus de la grippe, ou encore l'origine biologique des prions et les déterminants de la barrière d'espèce modulant leur capacité de transmission. Dans ce cas, il serait aisément concevable d'imaginer les mêmes travaux conduits dans des laboratoires de recherche ne relevant pas de la santé animale. Un positionnement santé animale présente néanmoins cer- tains atouts, notamment l'expertise pour des travaux expérimentaux lourds en zone confinée, et des liens privilégiés entretenus via des collaborations avec des scientifiques issus des champs de la pathogénie et de la génétique animale notamment.

La présente réflexion a été menée en relation avec les travaux de recherche en biologie humaine. Une démarche parallèle pourrait également être envisagée vis-à-vis des travaux réalisés en santé des plantes. On peut anticiper que cette analyse montrerait une certaine communauté d'outils et de méthodes avec la santé animale, un atout de biologie comparée, mais vraisemblablement peu d'enjeux partagés pour les agents pathogènes d'intérêt.

\section{3 / Réflexions sur l'organi- sation de la recherche en santé animale en France}

\section{1 / Structure de la recherche en santé animale}

La recherche publique française en santé animale, autour des enjeux présentés précédemment, est structurée au sein d'un ensemble d'établissements au nombre desquels figurent l'INRA, le CIRAD, l'ANSES ${ }^{10}$, les écoles vétérinaires, avec des Unités Mixtes de Recherche entre ces organismes. Quelques équipes appartiennent à d'autres organismes de recherche tels que le CNRS, le CEA, ou l'IRD. Pour certains, cette multiplicité des acteurs serait préjudiciable à une politique de recherche affirmée, à une recherche forte et organisée, et à une bonne lisibilité des recherches menées en santé animale, ce qui rejoint d'autres analyses sur l'organisation de la recherche publique ${ }^{11}$.

Les organismes de la recherche publique œuvrent aussi à la diffusion et au transfert de leurs résultats vers l'industrie (vaccins, diagnostics) et vers les filières de productions animales et agroalimentaires, pour mieux répondre à la demande sociétale. Vers l'industrie, audelà des collaborations scientifiques (thèses), le développement est générale- ment externalisé. Par exemple, le test de dépistage de l'ESB, initialement mis au point par des équipes du CEA et qui représente $80 \%$ des tests vendus dans le monde, a été développé et commercialisé par la société Bio-Rad. Pour les filières, la recherche se tourne vers les instituts et centres techniques, ou les organismes professionnels assurant l'encadrement agricole et le développement. L'accueil de leurs ingénieurs dans les laboratoires, à titre individuel ou dans le cadre d'un partenariat formalisé sous forme d'UMT (unité mixte technologique entre les instituts) ${ }^{12}$ facilite les collaborations, échanges, et transferts, ainsi que l'appropriation des outils et méthodes. Une fois validées et publiées, ces connaissances deviennent opérationnelles soit par l'intermédiaire d'experts scientifiques auprès des pouvoirs publics (missions d'expertise et d'appui scientifique et technique), soit par appropriation par les partenaires techniques, privés et publics, et application, qu'il s'agisse de plans collectifs de lutte, de plans de vaccination ou de prévention à l'échelle individuelle.

\section{2 / Financement et évaluation}

Les difficultés à faire financer et à «bien» publier (i.e. dans un périodique à facteur d'impact élevé) certains travaux de bonne qualité scientifique sont réelles dès lors qu'ils relèvent de santé animale pure sur des agents pathogènes non zoonotiques, en comparaison avec des travaux sur des agents pathogènes d'intérêt pour la santé publique. Ces difficultés semblent inversement proportionnelles à la généricité des connaissances produites et à leur intérêt biomédical potentiel. A titre d'exemple, un projet sur un agent pathogène non zoonotique, même excellent, sera systématiquement occulté par un projet abordant par exemple les fièvres hémorragiques dans un appel d'offres ANR 13 commun sur les maladies infectieuses, compte tenu des enjeux perçus par les évaluateurs. De la même façon, de nombreuses revues de santé humaine ou de disciplines scientifiques ayant un facteur d'impact plus élevé en raison de la taille beaucoup plus grande des communautés scientifiques concernées en biologie humaine par rapport à leurs équivalents en matière de santé animale,

\footnotetext{
10 L'ANSES résulte de la fusion de l'AFSSA avec l'AFSSET (Ordonnance n 2010-18 du 7 janvier 2010 portant création d'une agence nationale chargée de la sécurité sanitaire de l'alimentation, de l'environnement et du travail.

http://www.legifrance.gouv.fr/affichTexte.do?cidTexte=JORF-TEXT000021637409\&dateTexte=\&categorieLien=id. consulté le 30 avril 2010).

11 Comité national d'évaluation de la recherche, 2003. La recherche publique française : une évaluation. Rapport au Président de la république. La documentation française, Paris, $47 \mathrm{p}$.

12 Par exemple, l'ANSES et l'ITAVI ont créé l'UMT Sanivol (maîtrise de la santé des volailles), l'INRA et l'Institut de l'Elevage les UMT MSTB (maîtrise de la santé des troupeaux bovins) et UMT MSTPR (maîtrise de la santé des troupeaux de petits ruminants), et un ingénieur de R\&D des Groupements de défense sanitaire est accueilli dans une UMR INRA pour collaborer sur des thématiques de recherche concernant la prévention de maladies infectieuses et la certification (statut sanitaire) des cheptels.

13 Agence Nationale de la Recherche.
} 
accepteront très rarement un article sur des agents non zoonotiques qui, de fait, ne rentrent pas dans leur domaine.

Cet état de fait est extrêmement important à prendre en considération compte tenu de l'impératif actuel d'obtention de crédits pour financer les recherches, et de l'importance du critère «facteur d'impact» pour l'évaluation scientifique des équipes. Ce point est d'autant plus crucial que la proximité apparente des secteurs santé animale et biologie humaine ne rend pas pour autant leurs objectifs équivalents. Une approche un peu rapide de la question par des évaluateurs insuffisamment informés ou mal sensibilisés leur fera appliquer à des travaux en santé animale des critères et indicateurs appropriés au secteur de la biologie humaine, mais totalement inadaptés voire inéquitables dans le domaine de la santé animale. Une situation délicate est le cas d'unités de recherche qui traitent à la fois d'agents pathogènes zoonotiques et non zoonotiques. Au sein de la même unité, les équipes ne sont pas logées à la même enseigne du point de vue de la recherche de financements et du niveau de publication. Dans ce cas, des listes de revues disciplinaires considérées comme le top $15 \%$ ne suffisent pas à régler la question de la comparabilité des outils de mesure de la qualité de la production scientifique.

Il ressort de cette analyse qu'à qualité scientifique équivalente des travaux conduits, les recherches sur les maladies animales non zoonotiques sont moins facilement financées et moins bien publiées que les recherches sur les maladies animales zoonotiques. De la même façon, les recherches sur les maladies animales sont moins facilement financées et moins bien publiées que les recherches en biologie humaine. En l'absence d'action corrective spécifique, l'existence d'une «barrière des espèces» en matière de financement et de publication met donc en danger $80 \%$ des recherches en santé animale.

Il est donc absolument nécessaire, d'une part de militer très en amont de la construction des programmes ANR ou européens pour que des appels d'offre mentionnent spécifiquement des enjeux de santé animale, et d'autre part que l'INRA et son département Santé Animale affichent une politique de reconnaissance de ces enjeux et des productions scientifiques spécifiques qui y sont liées.

\section{3 / Recherche versus sur- veillance et contrôle des ma- ladies}

Un parallèle peut être fait entre le domaine de la recherche et celui de la surveillance et du contrôle des maladies. Il existe des courants de pensée à l'échelle internationale selon lesquels des économies d'échelle seraient possibles en regroupant, à l'échelle des pays, les services traitant de médecine vétérinaire avec ceux traitant de santé humaine. De même, les services publics comme la surveillance des maladies pourraient être considérés comme une variable d'ajustement sur laquelle agir pour faire des économies dans les pays endettés. Dans le même esprit, ces courants de pensée poussent aussi à ne considérer comme importants que les maladies animales à risque pour l'Homme, à cause de leur caractère zoonotique. Dans cette logique d'économies et de regroupement de la sphère des maladies animales avec celle de la santé humaine, les arbitrages financiers seraient naturellement en faveur des priorités de santé humaine, au détriment des services vétérinaires. La stratégie de l'OIE est le contrepied de cette position, considérant que la prévention est moins coûteuse que les crises, qu'une prévention de qualité repose sur des systèmes nationaux de santé animale assurant surveillance appropriée, détection précoce, transparence, et réaction rapide à des foyers de maladies animales, et sur un réseau pérenne de services vétérinaires dotés d'un budget spécifique. Aussi, en 2004, dans la résolution de l'assemblée mondiale des pays membres de l'OIE créant le Fonds mondial de l'OIE pour la santé et le bien-être des animaux, les 176 états membres de l'OIE ont déclaré les services vétérinaires bien public mondiall4. Les conséquences désastreuses des politiques de réduction des services publics, et l'efficacité de l'approche préventive et globale prônée par l'OIE, conduisent progressivement à un rééquilibrage des courants de pensée en faveur de cette approche. Ce changement est perceptible par exemple dans les documents internationaux mis en discussion lors des forums successifs sur le contrôle de l'Influenza aviaire ${ }^{15}$.

\section{4 / L'approche One world, one health}

L'approche One world, one health, ou «Un monde, une seule santé», comme le mentionne le Directeur de l'OIE dans un éditorial ${ }^{16}$, est indispensable dans le sens où «la prévention de tous ces nouveaux dangers (zoonotiques) repose sur une adaptation des dispositifs de gouvernance sanitaire au niveau global, régional et national sur un mode harmonisé et coordonné», mais «il n'est pas souhaitable que le concept "un monde, une seule santé" ouvre la voie à des initiatives dangereuses comme la recherche d'économies d'échelle basées sur des visions purement théoriques d'apprenti sorcier comme la fusion des Services vétérinaires et des Services de santé publique». Dans cette optique, il n'est en effet pas question de fusion des services, car chacun doit assumer sa fonction avec des moyens spécifiques et des approches particulières liées aux spécificités de sa mission, mais il est nécessaire de développer la collaboration, la coopération et les synergies. Il y a depuis quelques années consensus sur cette manière de voir de la part de l'OIE, de la FAO et de l'OMS. Différentes réflexions sont en cours pour définir des façons de mettre en œuvre cette coopération entre organisations ${ }^{16}$.

\section{5 / Parallèle avec l'industrie pharmaceutique}

La plupart des sociétés pharmaceutiques disposent de filiales dédiées à la santé animale, ce qui est lié au fait que les échelles économiques sont incomparables entre santé animale et santé humaine; à titre illustratif, le chiffre d'affaires d'un vaccin humain peut être 20 à 50 fois supérieur à celui d'un vaccin vétérinaire. Confronter dans un même arbitrage économique deux projets de vaccins très différents, même si chacun est a priori rentable, entraînerait automatiquement le choix du vaccin humain au détriment du vaccin vétérinaire. De la même manière, les services partagés seraient préférentiellement mis au service du projet de vaccin humain compte tenu de son enjeu autrement plus important. Enfin, il est aussi beaucoup plus difficile de chercher des financements publics, et par la même le complément en fonds privés pour aider au développement de vaccins contre des agents pathogènes non zoonotiques par rapport à des vaccins humains. Une fusion entre les activités humaines et animales se traduirait par la disparition du secteur animal, ou alors il ne serait développé que des modèles animaux ayant un intérêt direct pour l'Homme. Ce qui est commun aux vaccins animaux et humains est en revanche toute la technologie de production vaccinale,

\footnotetext{
14 http://www.oie.int/eng/oie/organisation/en vet serv.htm (consulté le 30 avril 2010).

$15 \mathrm{http}: / /$ www.oie.int/downld/FINAL CONCEPT NOTE Hanoi.pdf (consulté le 15 mai 2010).

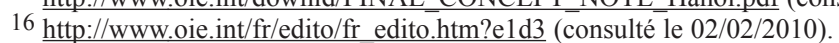


l'innovation dans ce domaine et la recherche en amont sur les familles d'agents pathogènes, la cytologie, certains aspects de l'immunologie, toutes connaissances qui méritent d'être mises en commun entre santé humaine et santé animale, sous la forme de coopération.

\section{Conclusions}

La présente réflexion, des avis d'experts et une lecture critique de la littérature conduisent à plusieurs constats :

- Les enjeux en santé animale, en dehors des maladies transmissibles à l'Homme (maladies zoonotiques), affirmés au premier chef par les instances internationales (OMS, FAO, OIE), sont énormes. Ils touchent à la sécurité alimentaire, à l'économie de l'agriculture et à l'ensemble des activités économiques qui en découlent, aussi bien dans les pays développés que dans les pays en voie de développement. Ces enjeux de santé animale hors maladies zoonotiques rejoignent par ailleurs des enjeux de santé publique et environnementaux, notamment sur les questions d'utilisation des xénobiotiques et le développement de l'antibiorésistance.

- Les spécificités de la recherche en santé animale sont d'ordre méthodologique et scientifique. Elles sont liées notamment aux particularités biologiques des espèces domestiques et à l'interaction entre l'Homme dans sa pratique de l'élevage et l'animal dans sa biologie et son évolution. La biologie animale n'a pas les mêmes questionnements scientifiques que la biologie humaine même lorsqu'elle traite des mêmes agents pathogènes, et elle s'enracine dans une réalité agricole et économique très spécifique. Rechercher un équilibre optimal entre rentabilité éco- nomique de l'exploitation, bien-être des animaux, maîtrise de la santé animale et qualité des produits d'origine animale implique, pour les acteurs de la santé animale, que ce soit du point de vue recherche ou développement, une connexion étroite avec les sciences de l'élevage et la profession agricole. Le métaprogramme Gestion Intégrée de la Santé Animale que lance l'INRA a pour objet de promouvoir une activité de recherche intégrée sur ce thème.

\section{- Les connaissances issues de la bio-} logie comparée sont nourries par les travaux conduits sur les espèces animales. Par exemple, leurs spécificités évolutives et dans certains cas leurs similitudes fonctionnelles avec l'Homme, font des modèles animaux une source de connaissances génériques. La diversité des espèces modèles étudiées et la maîtrise de modèles infectieux particuliers sont des atouts importants pour produire de telles connaissances sur le vivant.

Ces constats plaident fortement pour prendre en considération la singularité de la recherche en santé animale par rapport à la recherche en biologie humaine dans son organisation, son évaluation et son financement. Si tel n'était pas le cas, les priorités strictement biomédicales conduiraient à éliminer à plus ou moins brève échéance une recherche de qualité sur les maladies animales non zoonotiques. Un «traitement» particulier de cette recherche est donc nécessaire en termes d'enjeux; il doit passer par des appels d'offres spécifiques, par l'affirmation que son corpus de revues est différent de celui de la recherche biomédicale, et par l'évaluation de ses travaux à l'aune de ce corpus.

L'approche One health est importante dans la mesure où elle conduit à consi- dérer que la gestion de la santé nécessite une coordination renforcée entre le volet humain et le volet animal et, de la même manière, une collaboration approfondie entre recherche en biologie humaine et recherche en santé animale. L'organisation d'une telle collaboration ne peut que renforcer la capacité des uns et des autres à produire une science pertinente, et à potentialiser les efforts de recherche et les approches plus globales intégrant des composantes humaines et animales dans des projets fédérateurs.

En termes de recherche, cette collaboration peut prendre des formes variées et se situer à différents niveaux, allant de la coopération entre équipes jusqu'à l'organisation de la recherche et de son financement. Les questionnements en santé animale et biologie humaine sur un même agent pathogène zoonotique sont le plus souvent complémentaires. Ils permettent de bâtir des collaborations scientifiques pour répondre à des questions plus générales, et notamment aborder la complexité des systèmes biologiques de certaines maladies. Une autre forme est la mise en place d'appels d'offres de recherche communs entre santé publique et santé animale sur des agents pathogènes dont l'étude et le contrôle requièrent des approches de recherche combinées. Une illustration en est donnée par les travaux impulsés et financés par le Groupement d'Intérêt Scientifique sur les maladies à prions qui a permis d'aborder de manière pluridisciplinaire la question des encéphalopathies spongiformes transmissibles de l'Homme, des bovins (ESB) et des petits ruminants. A une échelle plus générale, la biologie comparée représente une précieuse source de connaissances.

\section{Références}

Assié S., Seegers H., Bouet J.M., Beaudeau F., 2001. Economic impact related to respiratory disorders of non-weaned calves in Charolais cow-calf farms in Pays de la Loire (France). $52^{\text {nd }}$ Meet. Eur. Ass. Anim. Prod., Budapest, Hongrie, 26-29 août 2001.

de Rycke J., 2009. World research landscape mapping of european research organisations. A bibliometrical analysis of research output (2004-2008). EMIDA ERA-NET - Rapport du Wordpackage 2, 60p. http://www.emidaera.net/upload/pdf/Final\%20WP2\%20report\% 20090407.pdf

de Rycke J., 2010. World mapping of research institutions, topics and collaboration in the field of infectious diseases of animals ; a bibliometric analysis using publication records extracted from the Web of Science (20062010), Report, 47p.
FAO, 2009. La situation mondiale de 1'alimentation et de l'agriculture: Le point sur l'élevage. Rome, Italie, 2009, 186p. http:/ /www.fao.org/docrep/012/i0680f/i0680f00.htm.

Fourichon C., 2001. Evaluation de l'impact zootechnique et économique des troubles de santé en élevage bovin laitier. Thèse, Ecole Nationale Supérieure Agronomique de Rennes, France, 252p.

Ganière J.P., André-Fontaine G., Drouin P., Faye B., Madec F., Rosner G., Fourichon C., Wang B., Tillon J.P., 1991. L'écopathologie : une méthode d'approche de la santé en élevage. INRA Prod. Anim., 4, 247-256.

Ganière J.P., 2004. Importance et hiérarchisation des zoonoses en France : le point de vue vétérinaire. Epidémiologie et Santé Animale, 46, 27-32.
Veissier I., Botreau R., Perny P., 2010. Evaluation multicrière appliquée au bien-être des animaux en ferme ou à l'abattoir : difficultés et solutions du projet Welfare Quality. Inra Prod. Anim., 23, 269-284.

WHO, 2002. Future trends in veterinary public health. Report of a WHO Study Group. 907. In: WHO Technical Reports Series, World Health Organization, Geneva,http://whqlibdoc.who.int/trs/ WHO_TRS_907.pdf. Consulté le 2010/11/17. 96p.

Williams R.B., 1999. A compartmentalised model for the estimation of the cost of coccidiosis to the world's chicken production industry. Int. J. Parasitol., 29, 8, 12091229. 


\title{
Remerciements
}

Les auteurs remercient vivement Thierry Pineau, chef du Département Santé Animale de l'INRA, qui a initié et soutenu la réflexion à l'origine de cet article, Claude Leclerc (Institut Pasteur), Alain Dehove, Elisabeth Erlacher-Vindel, Kasuaki Miyagishima (OIE), Jean-Christophe Audonnet, Michel Bublot, Catherine Charreyre, François Xavier Le Gros, Pascal Hudelet (Société Merial), pour leur contribution à cette réflexion collective, ainsi que Bernard Charley, Jean De Rycke, Michel Fougereau, Pierre Lekeux, Henri Salmon, Henri Seegers et Etienne Thiry pour leur lecture critique du rapport initial.

\section{Résumé}

Dans le contexte très évolutif de la recherche en santé animale, une réflexion sur ses enjeux, ses spécificités et ses synergies avec la recherche biomédicale, a été conduite à l'initiative de l'INRA. Affirmés au premier chef par l'OMS, la FAO et l'OIE, les enjeux en santé animale, hors des maladies transmissibles à l'Homme, sont énormes et touchent à la sécurité alimentaire, l'économie de l'agriculture et l'ensemble des activités économiques qui en découlent. S'y ajoutent les enjeux de santé publique (zoonoses, xénobiotiques, antibiorésistance), environnementaux et de bien-être animal.

La recherche en santé animale présente des spécificités d'ordre méthodologique et scientifique, liées notamment aux particularités biologiques des espèces domestiques et aux pratiques d'élevage. Elle n'a pas les mêmes questionnements scientifiques qu'en biologie humaine même lorsqu'elle traite des mêmes agents pathogènes et, connectée aux autres sciences animales (génétique, physiologie, zootechnie), elle s'enracine dans une réalité agricole et économique très spécifique.

Des synergies génériques et méthodologiques existent néanmoins avec la recherche biomédicale, en particulier autour des outils et des modèles biologiques. Certaines espèces domestiques (tel le porc) présentent en outre des similitudes fonctionnelles avec l'Homme, plus que le rongeur de laboratoire.

Ainsi la singularité de la recherche en santé animale par rapport à la recherche en biologie humaine devrait être prise en compte dans son organisation, son évaluation et son financement, via une politique de reconnaissance des enjeux spécifiques. Simultanément, l'approche one health devrait faciliter une collaboration approfondie entre recherche en biologie humaine et recherche en santé animale, à l'échelle des équipes ou des programmes.

\begin{abstract}
Issues and special features of research on animal health

In the moving context of research on animal health, a collective discussion has been carried out on its issues, special features and synergism with biomedical research. Strongly enhanced by WHO, FAO and OIE, the issues of animal health are important and deal with food security, agriculture economics and all economical activities that follow from it. Others are related to public health (zoonoses, xenobiotics, antimicrobial resistance), environment and animal welfare.

Research on animal health has got special features concerning methodology and scientific questions, among others the particular biological nature of domestic species and breeding practices. Scientific questions are not similar to those of biomedical research, even if dealing with the same pathogens; connected to the other animal sciences (genetics, physiology, animal breeding), research on animal health takes root in a very specific agricultural and economical reality.

Nevertheless, generic and methodological synergies do exist with biomedical research, particularly concerning biological tools and models. Some domestic species like pig have functional similarities with Humans, better than with mouse or rat.

Thus, the peculiarity of research on animal health compared to biomedical research should be considered in its organisation, evaluation and funding, through a political recognition of specific issues. Simultaneously, the one health approach should facilitate a thorough collaboration between biomedical research and research on animal health, at the levels of research teams and research programmes.
\end{abstract}

DUCROT C., BED'HOM B., BERINGUE V., COULON J.-B., FOURICHON C., GUÉRIN J.-L., KREBS S., RAINARD P., SARRADIN P., SCHWARTZ I., TORNY D., VAYSSIER-TAUSSAT M.-L., ZIENTARA S., ZUNDEL E., 2010. Enjeux et spécificités de la recherche en santé animale. Inra Prod. Anim., 23, 359-368. 